\title{
Progress in the diagnosis and treatment of COVID-19 and the role of surgeons in the front line of the pandemic
}

\author{
Yun-Jie Shi ${ }^{2} \cdot$ Hao Wang ${ }^{1,2}$
}

Received: 21 April 2020 / Accepted: 11 July 2020 / Published online: 4 September 2020

(c) Springer Nature Singapore Pte Ltd. 2020

\begin{abstract}
The current struggle to control and contain COVID-19 is critical and surgeons are on the front line in the fight against this virus. Surgeons, and other medical workers in the field of surgery, have a solid foundation and experience in medical treatment and intensive care, and an understanding of the support of respiratory, circulatory, digestive, and other systemic organs. Furthermore, the operative standards of aseptic technique in their daily work enable surgeons to adapt to the working environment in infected areas. As surgeons in the anti-pandemic front line in China, we describe our experience with the diagnosis and treatment of COVID-19 in this country and how the work of surgeons is unfolding during the pandemic.
\end{abstract}

Keywords Surgeons · Anti-COVID-19 · Front line

\section{Introduction}

The COVID-19 pandemic has spread globally and the situation is grim. COVID-19 is concealed and highly contagious, and can be transmitted through droplets, contacts, the fecal-oral route, and aerosols. According to the International Code for Virus Classification and Nomenclature of the International Committee on Taxonomy of Viruses (ICTV), the virus was initially named "severe acute respiratory syndrome coronavirus 2" (SARS-Cov-2) [1]. The key factors for success in the fight against COVID-19 include accurate screening and isolation of infected patients, availability and appropriate allocation of adequate medical resources, and correct treatment of critically ill patients. Surgeons also play a pivotal role in this epidemic.

Hao Wang

wanghaohh@vip.126.com

1 Ward 1, Department (2) of Infectious Diseases, Huoshenshan Hospital, Wuhan 430113, China

2 Department of Colorectal Surgery, Changhai Hospital, Naval Medical University (Second Military Medical University), \#168, Changhai Road, Shanghai 200433, China

\section{Progress in the diagnosis and treatment in CoVID-19 in China}

\section{Progress in COVID-19 diagnosis}

The diagnosis of COVID-19 is based on epidemiological history, symptoms and signs, blood tests, chest imaging scans, and nucleic acid or serum antibody detection. Chest computed tomography (CT) is also an important examination for COVID-19 patients because of its simplicity and non-invasiveness. The diagnostic sensitivity of chest CT to COVID-19 is reported to be higher than that of the real-time polymerase chain reaction (RT-PCR) nucleic acid test, especially during the incubation period before a positive result of the RT-PCR nucleic acid test is detectable, when some characteristic features of COVID-19 have been observable on CT images [2]. Most lesions are distributed in the subpleural or lateral lung regions of both lower lungs, and some along the bronchovascular bundle [3]. The typical CT presentation of COVID-19 is ground glass opacity (GGO) shadows, pulmonary consolidation, and pulmonary sub-segment vasodilation to a vascular diameter of greater than $3 \mathrm{~mm}$ [4]. The artificial intelligence (AI) model combined with the CT imaging and clinical information has the same diagnostic accuracy as a senior chest radiologist and, therefore, serves as a useful screening tool [5]. A multi-center retrospective study found that extensive learning of the AI model can help detect COVID-19 accurately and distinguish it from 
community-acquired pneumonia and other lung diseases [6]. RT-PCR detection of SARS-Cov-2 nucleic acid and the expression of specific antibodies against SARS-Cov-2 in serum is a common diagnostic technique; however, there have been false-negative results of SARS-Cov- 2 nucleic acid detection, which may even cause a re-detectable positive RNA test in discharged patients. Therefore, it is necessary to continuously optimize the various techniques of sampling, transferring, and testing to ensure the accuracy of the confirmed diagnosis. Studies have shown that the nucleic acid positive rate of nasal swab sampling is higher than that of throat swab sampling [7]. We collected a total of 122 nasal, 114 throat, and 97 rectal swabs from 44 recovering patients. Comparison of the positive rates of viral nucleic acid detection showed that the nose-throat double-swab sampling was the best clinical sampling method, especially as one of the discharge criteria for recovering patients (unpublished data).

Asymptomatic infection means no presenting clinical symptoms, but a SARS-Cov-2 pathogenic test or a serumspecific antibody test of respiratory tract specimens shows a positive result [8]. There are two common situations: one is that the infection may be in the incubation period and a positive nucleic acid test result can only be detected in the middle and late periods; the other is that the symptoms are mild and a positive nucleic acid test result can only be detected during convalescence. It is well documented that droplets are the main mode of transmission of asymptomatic infections [9]. Currently, reports about patients with asymptomatic infection are mainly identified during the screening of close contacts. Such a limited research method will inevitably underestimate the number of patients with asymptomatic infection, and more importantly, asymptomatic patients are no less contagious [8]. Therefore, we should devote more effort to screening and identifying such patients as early as possible, especially those who are scheduled to undergo surgery. From May 11, 2020, Wuhan City, China began screening for asymptomatic infections by universal nucleic acid detection in a "combined sample" mode. At the same time, the results of the Phase-1 Clinical Trial of the Ad5 adenovirus vector COVID-19 vaccine (Ad5-nCoV) published by Academician Chen Wei's team at the Chinese Academy of Military Medical Sciences on May 22, 2020 showed that the vaccine was safe and well tolerated [10].

\section{Progress in COVID-19 treatment}

COVID-19 can progress rapidly in some patients, causing them to be severely or critically ill within days. The typical clinical manifestations of critically ill patients are respiratory failure and even multiple organs failure. A multi-discipline team (MDT) is required for the emergency treatment of such patients, including tracheal intubation or tracheotomy, ventilator-assisted breathing, ECMO, hemodialysis, and artificial liver blood purification. Progressive exacerbation of the condition is associated with a continuous decrease in the number of lymphocytes and a significant increase in neutrophils [11]. According to Professor Taisheng Li [12, 13], this situation may arise from an "inflammatory storm". To curb this reaction as quickly as possible, these patients should be given a sufficient amount of immunoglobulin $(0.3-0.5 \mathrm{~g} / \mathrm{kg} / \mathrm{day})$ in the early stage of acute exacerbation, about 7 days after the onset of disease. Studies have shown that there is a correlation between inflammatory factor IL-6 and the severity of COVID-19 infection. The IL6 antagonist tocilizumab (400-800 $\mathrm{mg}$ ) can also block the "inflammatory storm" and ameliorate the symptoms of moderate and severe COVID-19 [14]. Although administering a plasma infusion to critically ill patients during the recovery period can relieve their clinical symptoms within $24-48 \mathrm{~h}$, it cannot reduce the mortality rate of critical and end-stage COVID19 , and therefore the treatment should be started as early as possible [15]. The therapeutic effect of stem cell infusion against severe COVID-19 is also remarkable [16], knowing that it can reduce or even reverse the damage arising from interstitial pneumonia through paracrine, anti-inflammatory, and immune regulations and antioxidant mechanisms. The inhalation of a mixture of hydrogen and oxygen can significantly improve breathing difficulties in most COVID19 patients [17]. Moreover, a hypercoagulable state may develop in some patients because SARS-Cov-2 also attacks vascular endothelial cells. Abnormal coagulation caused by excessive immune response can lead to significant changes in coagulation parameters, or even disseminated intravascular coagulation (DIC) in critically ill patients [12]. The subcutaneous administration of low molecular heparin $(100 \mathrm{u} /$ $\mathrm{kg}, \mathrm{q} 12 \mathrm{~h}$ ) is given to prevent deep vein thrombosis [12]. At the same time, alterations in the intestinal flora through the oral administration of probiotics may also alleviate the severity of the disease [18]. All these treatment measures have been applied in our ward, and achieved good therapeutic outcomes.

The symptoms were relieved by appropriate treatment in some COVID-19 patients, but the result of their repeated nucleic acid amplification tests (NAATs) remained positive. The possible reasons for this are the pathogenic characteristics of SARS-Cov-2, immunodeficiency of the patient, systemic use of glucocorticoids, intermittent detoxification of the body during the process of inflammatory absorption, and problems related to the etiological detection methods [19]. For such patients, it is necessary to intensify systemic support, enhance immunity, actively treat the underlying diseases, and administer antiviral treatment. Recent studies suggest that some drugs, including hydroxychloroquine [20] and certain Chinese herbal medicines [21], have the potential to treat COVID-19 with promising application prospects. On May 19, 2020, the South Korean CDC [22] released a 
study on patients with COVID-19 re-infection, suggesting that COVID-19 patients with re-infection are not infectious. Patients discharged from hospital after two negative nucleic acid test results do not need to isolate for 14 days.

\section{Surgeons play an important role in the front-line battle against COVID-19}

\section{The unique role of surgeons in the battle against COVID-19}

Surgeons have vast experience in perioperative management and can treat COVID-19 patients independently according to their distinguishing features. Pulmonary infection is a common and potentially devastating complication that surgeons encounter regularly, especially in elderly surgical patients. It is often similar to the severe pneumonia associated with COVID-19 and the treatment principles and experience can be shared. The treatments for ARDS caused by severe and end-stage pneumonia are the same [23]. Moreover, surgeons are familiar with the process of MDT and can adapt to this collaborative mode of work quickly in the front-line battle against COVID-19 [24]. In areas where there are large numbers of patients with COVID-19 infection, there is often a shortage of personal protective equipment, and therefore reducing the in-and-out frequency of surgeons who need to work long hours in isolation wards can help ease the burden of supply shortage. At the same time, surgeons can consult about procedure-related complications of infected patients in the COVID-19 wards [25]. Surgical complications in COVID-19 wards should be treated primarily with non-surgical measures. If the non-surgical measures do not work well, surgeons should choose the method that will cause the least body fluid exposure and minimal trauma to the patient, such as colonoscopic interventions that colorectal surgeons can perform easily, and avoid surgery as much as possible [26].

\section{What surgeons must learn in the front-line battle against COVID-19?}

Being familiar with the characteristic features of COVID-19 and the related diagnosis and treatment are paramount for surgeons working in the front-line battle against COVID19. Surgeons need to acquire more specialized knowledge in internal medicine, infectious diseases, and intensive care medicine. They should also master related rescue skills for critical COVID-19 patients within the shortest possible time, including tracheal intubation in emergency situations and the use of ventilators or ECMO [27]. At the same time, it is essential for surgeons to be adept at donning and doffing personal protective equipment (PPE) and to understand the requirements of working in the isolation ward [28]. Only when surgeons meet all these requirements can they apply rational and scientific rescue measures to the treatment of each COVID-19 and critically ill patient.

\section{Perioperative management and standardization of surgical procedures for COVID-19 patients}

\section{Perioperative management of COVID-19 patients}

The surgeon must screen all "non-COVID-19" patients. Most hospitals in China have established fever clinics to screen patients with confirmed or suspected infections in advance, which can effectively triage infected and noninfected patients and reduce the exposure of surgical staff to COVID-19 infection [29]. In principle, scheduled or elective surgery should be postponed for patients with suspected or confirmed COVID-19. Surgery should be performed only for life-threatening emergencies in these patients. To identify the appropriate timing of emergency surgery for a critically ill COVID-19 patient, MDT discussion should take place. Patients with suspected or confirmed COVID-19 infection who require emergency surgery must be transferred to the negative-pressure operating room ( -5 pa or less) by the designated person via a dedicated channel [30]. The number of medical staff involved in the surgery must be strictly limited (5-6 people). For critically ill patients, the anesthesiologists should arrange for ICU equipped with isolation measures. All surgical participants should follow third-level protection and adhere to 14-day quarantine for medical observation after the operation [31]. All surgical waste must be sorted and disposed of immediately in a double-layer clinical waste bag marked "COVID-19". In addition to routine sterilization of the surfaces and floors of the operating room, equipment purification maintenance engineers should be notified to replace the air inlet and exhaust systems and the internal filter of the purification unit. Patients must be isolated in an isolation ward after surgery. Apart from the surgical management, it is also necessary to monitor major vital signs and blood oxygen saturation routinely, administer oxygen and perform regular chest CT scans.

\section{Surgical specifications for COVID-19 patients}

In addition to routine preoperative evaluation, anesthesiologists must monitor every patient for signs of lung infection and their general condition. Before the end of operation, the anesthesiologist should clear the patient's airway secretions under deep anesthesia and remove the tracheal intubation before the patient wakes up [32]. Surgical instruments, for open surgery or endoscopic surgery, must be cleaned after 
contact with the patient's body fluids. Aerosols are produced in the process of cutting or separating tissues and organs during surgery. The smoke evacuation system with appropriate filters can reduce the risk of exposure [33]. The power of the energy platform should be minimized and the prolonged use of electrical and ultrasound equipment to cut the same anatomical site avoided. During laparoscopic surgery, $\mathrm{CO}_{2}$ circulation in the abdomen will accelerate aerosol diffusion to the air; therefore, it is necessary to check meticulously for air leakage around the trocar hole before laparoscopic surgery. It is recommended to use a constant-pressure pneumoperitoneum machine and a suction device to remove smoke and fog before opening the abdomen [34]. It is also necessary to follow the indication for ostomy during surgery. In pandemic focus areas, the nursing process after fistula surgery can increase the risk of infection of the MCWs. Finally, the surgeon needs to wear robust protective equipment and multi-layer gloves, which do increase the difficulty of the operation, but can be adapted to through short-term training.

\section{Conclusion}

Many surgeons have participated in the anti-COVID-19 work in China, which has helped relieve the current shortage of front-line MCWs, although it is their responsibility to do so. As surgeons have diverse clinical experience, their participation in the diagnosis and treatment of COVID-19 patients will help control the pandemic provided they utilize their unique advantages and close the gap with the infection control workers.

Funding This work was supported by Bethune Medical Science Research Fund of Beijing Bethune Charitable Foundation (Grant No. SG055CS, 2020).

\section{Compliance with ethical standards}

Conflict of interest We have no competing interests to declare.

\section{References}

1. Cascella M, Rajnik M, Cuomo A, Dulebohn SC, Di Napoli R. Features, evaluation and treatment coronavirus (COVID-19). StatPearls. Treasure Island (FL): StatPearls Publishing Copyright ${ }^{\odot}$ 2020, StatPearls Publishing LLC; 2020.

2. Tao A, Zhenlu Y, Hongyan H, Chenao Z, Chong C, Wenzhi L, et al. Correlation of chest CT and RT-PCR testing in coronavirus disease 2019 (COVID-19) in China: a report of 1014 cases. Radiology. 2020; Online ahead of print.

3. Xie X, Zhong Z, Zhao W, Zheng C, Wang F, Liu J. Chest CT for typical 2019-nCoV pneumonia: relationship to negative RT-PCR testing. Radiology. 2020;2020:200343.
4. Damiano C, Michela P, Francesco P, Tiziano P, Carlotta R, Gisella G, et al. Chest CT features of COVID-19 in Rome, Italy. Radiology. 2020; Online ahead of print.

5. Mei X, Lee HC, Diao KY, Huang M, Lin B, Liu C, et al. Artificial intelligence-enabled rapid diagnosis of patients with COVID-19. Nat Med. 2020;19:1-5.

6. Lin L, Qin L, Zeguo X, Youbing Y, Xin W, Bin K, Junjie B, et al. Artificial intelligence distinguishes COVID-19 from community acquired pneumonia on Chest CT. Radiology. 2020; Online ahead of print.

7. Sungnak W, Huang N, Becavin C, Berg M, Queen R, Litvinukova M, et al. SARS-CoV-2 entry factors are highly expressed in nasal epithelial cells together with innate immune genes. Nat Med. 2020;26(5):681-7.

8. Qiu J. Covert coronavirus infections could be seeding new outbreaks. Nature. 2020.

9. Xu J, Li Y, Gan F, Du Y, Yao Y. Salivary glands: potential reservoirs for COVID-19 asymptomatic infection. J Dent Res. 2020:22034520918518.

10. Zhu F-C, Li Y-H, Guan X-H, Hou L-H, Wang W-J, Li J-X, et al. Safety, tolerability, and immunogenicity of a recombinant adenovirus type-5 vectored COVID-19 vaccine: a dose-escalation, open-label, non-randomised, first-in-human trial. The Lancet. 2020.

11. Qin C, Zhou L, Hu Z, Zhang S, Yang S, Tao Y, et al. Dysregulation of immune response in patients with COVID-19 in Wuhan, China. Clin Infect Dis. 2020.

12. Cao W, Li T. COVID-19: towards understanding of pathogenesis. Cell Res. 2020;30(5):367-9.

13. Lin L, Lu L, Cao W, Li T. Hypothesis for potential pathogenesis of SARS-CoV-2 infection-a review of immune changes in patients with viral pneumonia. Emerg Microbes Infect. 2020;9(1):727-32.

14. Alzghari SK, Acuna VS. Supportive treatment with tocilizumab for COVID-19: a systematic review. J Clin Virol. 2020;127:104380.

15. Zeng QL, Yu ZJ, Gou JJ, Li GM, Ma SH, Zhang GF, et al. Effect of convalescent plasma therapy on viral shedding and survival in COVID-19 patients. J Infect Dis. 2020.

16. Zhang C, Shi L, Wang FS. Liver injury in COVID-19: management and challenges. Lancet Gastroenterol Hepatol. 2020;5(5):428-30.

17. Guan WJ, Chen RC, Zhong NS. Strategies for the prevention and management of coronavirus disease 2019. Eur Respir J. 2020; $55(4)$.

18. Zuo T, Zhang F, Lui GCY, Yeoh YK, Li AYL, Zhan H, et al. Alterations in gut microbiota of patients with COVID-19 during time of hospitalization. Gastroenterology. 2020.

19. Zhou L, Liu K, Liu HG. Cause analysis and treatment strategies of "recurrence" with novel coronavirus pneumonia (COVID-19) patients after discharge from hospital. Chin J Tuberc Respir Dis. 2020;43(4):281-4.

20. Gao J, Tian Z, Yang X. Breakthrough: chloroquine phosphate has shown apparent efficacy in treatment of COVID-19 associated pneumonia in clinical studies. Biosci Trends. 2020;14(1):72-3.

21. Yu S, Wang J, Shen H. Network pharmacology-based analysis of the role of traditional Chinese herbal medicines in the treatment of COVID-19. Ann Palliat Med. 2020;9(2):437-46.

22. Korea Centers for Disease Control and Prevention. Findings from investigation and analysis of re-positive cases. 2020. https://www.cdc.go.kr/board/board.es? mid=a304020000 $00 \&$ bid $=0030 \&$ act $=$ view \&list_no $=367267 \&$ nPage $=1 \#$ a . Accessed 19 May 2020.

23. Ahn DG, Shin HJ, Kim MH, Lee S, Kim HS, Myoung J, et al. Current status of epidemiology, diagnosis, therapeutics, and vaccines for novel coronavirus disease 2019 (COVID-19). J Microbiol Biotechnol. 2020;30(3):313-24. 
24. Broderick D, Kyzas P, Baldwin AJ, Graham RM, Duncan T, Chaintoutis C, et al. Surgical tracheostomies in COVID-19 patients: a multidisciplinary approach and lessons learned. Oral Oncol. 2020;2020:104767.

25. Mojoli F, Mongodi S, Orlando A, Arisi E, Pozzi M, Civardi L, et al. Our recommendations for acute management of COVID-19. Critical Care (London, England). 2020;24(1):207.

26. Goh SSN, Yeo DXW, Kaushal SA, Yeong HK, Tan GWL. Surgical and endoscopy protocols for general surgeons during the COVID19 pandemic: an institutional experience in Singapore. Br J Surg. 2020.

27. Shen C, Wang Z, Zhao F, Yang Y, Li J, Yuan J, et al. Treatment of 5 critically ill patients with COVID-19 with convalescent plasma. JAMA. 2020.

28. Cook TM. Personal protective equipment during the coronavirus disease (COVID) 2019 pandemic: a narrative review. Anaesthesia. 2020.

29. Zhang J, Zhou L, Yang Y, Peng W, Wang W, Chen X. Therapeutic and triage strategies for 2019 novel coronavirus disease in fever clinics. Lancet Respir Med. 2020;8(3):e11-e12.

30. De Simone B, Chouillard E, Di Saverio S, Pagani L, Sartelli M, Biffl WL, et al. Emergency surgery during the COVID-19 pandemic: what you need to know for practice. Ann R Coll Surg Engl. 2020;102(5):323-32.

31. Ya-Li C, Shu-Li Z, Zeng-Mei Z, Zhi-Hui S, Hai-Yun Z, et al. Management strategies and suggestions for operating room for emergency operations on novel coronavirus pneumonia patients. J Xi' an Jiaotong Univ (Medical Sciences). 2020.

32. Coccolini F, Perrone G, Chiarugi M, Di Marzo F, Ansaloni L, Scandroglio I, et al. Surgery in COVID-19 patients: operational directives. World J Emerg Surg. 2020;15(1):25.

33. Zheng MH, Boni L, Fingerhut A. Minimally invasive surgery and the novel coronavirus outbreak: lessons learned in China and Italy. Ann Surg. 2020.

34. Liu Z, Ding Z, Guan X, Zhang Y, Wang X, Khan JS. Optimizing response in surgical systems during and after COVID-19 pandemic: lessons from China and the UK-perspective. Int J Surg. 2020;78:156-9.

Publisher's Note Springer Nature remains neutral with regard to jurisdictional claims in published maps and institutional affiliations. 